DE

M E D I C I N A

T R O P I C A L

$\mathrm{DE}$

SÃO PAULO

JOURNAL OF THE SÃO PAULO INSTITUTE OF TROPICAL MEDICINE

(1) Universidade Federal Fluminense,

Faculdade de Medicina Veterinária, Niterói, Rio de Janeiro, Brazil

(2) Universidade Federal Fluminense, Instituto Biomédico, Departamento de Microbiologia e Parasitologia, Niterói, Rio de Janeiro, Brazil

(3) Universidade Federal Fluminense, Faculdade de Veterinária, Departamento de Clinica e Cirurgia, Niterói, Rio de Janeiro, Brazil

Correspondence to: Flávia Fernandes de Mendonça Uchôa

Universidade Federal Fluminense,

Faculdade de Medicina Veterinária, Rua

Vital Brasil Filho, 64, CEP 24230-340, Vital

Brasil, Niterói, RJ, Brazil

Tel: +5521999188136

Fax: + 552126094411

E-mail: flavia_uchoa@yahoo.com.br

Received: 28 November 2016

Accepted: 31 May 2017

\section{The influence of serial fecal sampling on the diagnosis of giardiasis in humans, dogs, and cats}

Flávia Fernandes de Mendonça Uchôa ${ }^{1}$, Adriana Pittella Sudré2 ${ }^{2}$ Daniel de Barros Macieira ${ }^{3}$, Nádia Regina Pereira Almosny ${ }^{3}$

\section{ABSTRACT}

Giardia infection is a common clinical problem in humans and pets. The diagnosis of giardiasis is challenging as hosts intermittently excrete protozoan cysts in their feces. In the present study, we comparatively evaluated two methods of serial fecal sampling in humans, dogs, and cats from Rio de Janeiro, Brazil. The Faust et al. technique was used to examine fecal specimens collected in triplicate from 133 patients (52 humans, 60 dogs, and 21 cats). Specimens from 74 patients were received from the group assigned to carry out sampling on consecutive days - 34 humans, 35 dogs, and 5 cats, and specimens from 59 patients were received from the group assigned to carry out sampling on non-consecutive, separate days - 18 human beings, 25 dogs, and 16 cats. G. duodenalis cysts were found in stools of 30 individuals. Multiple stool sampling resulted in an increase in the number of samples that were positive for Giardia in both groups. The authors therefore conclude that multiple stool sampling increases the sensitivity of the Faust et al. technique to detect $G$. duodenalis cysts in samples from humans, cats and dogs.

KEYWORDS: Giardia duodenalis. Fecal examination. Protozoa. Cysts.

\section{INTRODUCTION}

Giardia duodenalis is a flagellated protozoan parasite that infects millions of hosts around the world ${ }^{1}$ causing intestinal disease in humans, companion animals and livestock ${ }^{2-4}$. Giardia cysts are excreted intermittently for indefinite periods of time in the feces of both symptomatic patients and asymptomatic carriers from different species, such as humans, dogs and ruminants ${ }^{5,6}$, and can remain viable and infective in the environment for several months, which contributes to the persistence and dispersion of this pathogen ${ }^{7,8}$. In subclinical infections, asymptomatic carriers can shed Giardia cysts in their stools for longer periods, which favors reinfection and persistent environmental contamination ${ }^{9}$.

In recent years there has been a growing interest in the zoonotic and epidemic potential of Giardia sp. prompting greater demand for diagnostic tools ${ }^{10}$. Microscopic examination of fecal samples is effective and cheap, and is the most common test used to diagnose giardiasis ${ }^{11,12}$. Besides giardiasis, a large variety of helminth and protozoan infections can be detected by fecal examination ${ }^{13,14}$. However, the use of a single zinc sulfate flotation test in a stool sample may be insufficient to make the diagnosis of Giardia sp. infection since cyst shedding in feces is intermittent ${ }^{15-22}$. Therefore, the diagnosis of giardiasis presents a challenge to parasitologists ${ }^{23}$. The disease therefore may be underdiagnosed as intermittent cyst shedding can lead to a false negative result especially if only one fecal sample is examined. It is, therefore, 
advisable to test multiple fecal samples in order to increase the probability of getting a positive result for Giardia and avoid false negative results ${ }^{19,24}$.

Given the importance of using classical parasitological methods for the diagnosis of parasitic diseases, the aim of this study was to comparatively assess two methods of serial fecal sampling, increasing the sensitivity in the diagnosis of G. duodenalis infection. Moreover, samples were collected from humans, dogs, and cats from the East Metropolitan area of Rio de Janeiro State, Southeast Brazil, to compare the efficiency of both sampling methods for each host, aiming to confirm previous studies in humans and dogs using a different methodology, and to perform the first study assessing the influence of serial sampling on feline hosts.

\section{MATERIALS AND METHODS}

In the present study, two methods of serial fecal sampling were comparatively assessed; multiple fecal samples from humans, dogs, and cats living in the East Metropolitan area of Rio de Janeiro State, Southeast Brazil, were collected between June and October 2015. The population was randomly selected among pet owners attended in veterinary clinics and veterinary clinic laboratories of Niterói. Nine hundred stool sample kits were manufactured and distributed among the individuals who volunteered to participate in this study.

The inclusion criteria for participants were the signature of a consent term and the correct collection of three fecal samples. Participants collected fecal specimens from human beings, dogs, and cats living in the city of Niterói, Rio de Janeiro State (RJ), Southeast Brazil. The cats and dogs were owned pet-animals and their feces were picked up from soil, and were collected fresh after expulsion. In addition, the volunteers who collected their feces were advised about the importance of collecting samples of the same dog/cat day by day.

Each stool sample kit consisted of 3 sampling bottles and instructions for sample collection, handling, and storage.

Two groups were formed for the collection of fecal samples from the three aforementioned species, and different sampling timings were used. Participants were assigned to each of these groups based on the random distribution of the stool sample kits among the volunteers. Half of the forms with instructions for sample collection informed participants to collect 3 fecal samples in consecutive days (daily sampling). In the other half participants were asked to collect 3 fecal samples every other day (every-otherday sampling). No preservation media were used for the storage of stool samples; these fecal specimens were kept refrigerated for a maximum of $24 \mathrm{~h}$.
Fecal aliquots were analyzed using the Faust et al..$^{25}$ technique for the diagnosis of giardiasis. Approximately $4 \mathrm{~g}$ of faeces were diluted in $20 \mathrm{~mL}$ of distilled water in order to obtain a homogeneous solution. After that, the samples were strained through a wire gauze onto a centrifuge glass tube and submitted to centrifugation for $2 \mathrm{~min}$ at $640 \mathrm{~g}$. That washing step was performed twice, discarding the supernatant. Subsequently the sediment was re-suspended with $7 \mathrm{~mL}$ of zinc sulfate solution (sp gravity 1.200 ). The flotation was performed with centrifugation for $2 \mathrm{~min}$ at $640 \mathrm{~g}$. Five loops of the convex meniscus were collected with a bacteriological loop, as recommended by Pereira and Ferreira ${ }^{26}$. They were placed on a microscope slide, stained with a drop of Lugol's iodine and covered with a coverslip.

The same trained clinical pathologist performed both sample preparation and microscopic examination. Each sample was numbered to allow a blind exam. In order to increase the sensitivity of the fecal flotation and improve the diagnosis of giardiasis, two slides were prepared from each sample for microscopic examination. The slides were observed at 100X magnification. To confirm the presence of Giardia cysts, a 400X magnification was used. Microscopic examination was performed up to $24 \mathrm{~h}$ after sample collection.

The results obtained with each of the two sampling schemes in all three species were compared using the Fisher exact test for independent variables and statistical significance was determined based on a 2-sided p-value of 0.05 . The cumulative frequency of positivity was calculated considering one-, two- or three-day sampling for each host in each sampling scheme. The Fisher exact test was used to compare the calculated frequencies.

\section{ETHICS}

This study was approved by the Research Ethics Committee of the School of Medicine/Antonio Pedro University Hospital (CAAE 44055615.0.0000.5243) and by the Ethics Committee on Animal Use from the Fluminense Federal University (UFF) ( $\mathrm{N}^{\circ}$ 643), Niterói, RJ, Brazil.

\section{RESULTS}

A total of 415 stool samples from 141 individuals were received for analysis ( 58 humans, 62 dogs, and 21 cats). In the daily sampling group, 74 stool samples from humans $(n=34), \operatorname{dogs}(n=35)$, and cats $(n=5)$ were obtained. In the every-other-day sampling group, 59 stool samples from humans $(n=18), \operatorname{dogs}(n=25)$, and cats $(n=16)$ were obtained. Eight participants did not collect all three stool samples as requested, and they were excluded from the analysis. 
G. duodenalis cysts were detected on fecal samples from 30/133 patients $(22.6 \%)$ evaluated by the Faust technique. In the daily sample collection scheme, 7/35 (20\%) dogs and $3 / 5(60 \%)$ cats were diagnosed. In the every-other-day scheme, it was possible to identify $5 / 25$ positive (20\%) dogs, $4 / 16$ (25\%) positive cats and 7/18 (38.9\%) positive humans. The results obtained in each sampling scheme and for each animal species are shown in Table 1. Fecal samples from some individuals were Giardia-negative in the first fecal examination and Giardia-positive in the second or third fecal examination.

Table 1 - Number of Giardia duodenalis-positive stool samples in each sampling scheme and in each animal species analyzed (humans, dogs, and cats). The Faust technique ${ }^{1}$ was used to screen fecal specimens

\begin{tabular}{lcc}
\hline & \multicolumn{2}{c}{ Giardia-positive samples } \\
\cline { 2 - 3 } & Daily sampling & $\begin{array}{c}\text { Every-other-day } \\
\text { sampling }\end{array}$ \\
\hline Humans & $0 / 34(0 \%)$ & $7 / 18(38.9 \%)$ \\
Dogs & $7 / 35(20 \%)$ & $5 / 25(20 \%)$ \\
Cats & $3 / 5(60 \%)$ & $4 / 16(25 \%)$ \\
All three species & $10 / 74(13.5 \%)$ & $16 / 59(27.1 \%)$ \\
\hline
\end{tabular}

Serial stool sample collection increased the number of Giardia-positive fecal specimens in both, daily sampling and every-other-day sampling schemes (Figures 1 and 2 and Table 2).

When considering samples from humans regardless of the sampling group, there was an increase of 2 Giardiapositive patients with a two days sampling, an increment of $28.6 \%(2 / 7)$ in the detection of carriers of this protozoan infection $(\mathrm{p}=0.46)$. Since no human host from daily sampling group was positive, it was not possible to compare both groups for this host.

When results from dogs regardless of the sampling group were analyzed, we found that collection of two samples allowed the diagnose of 2 extra positive patients

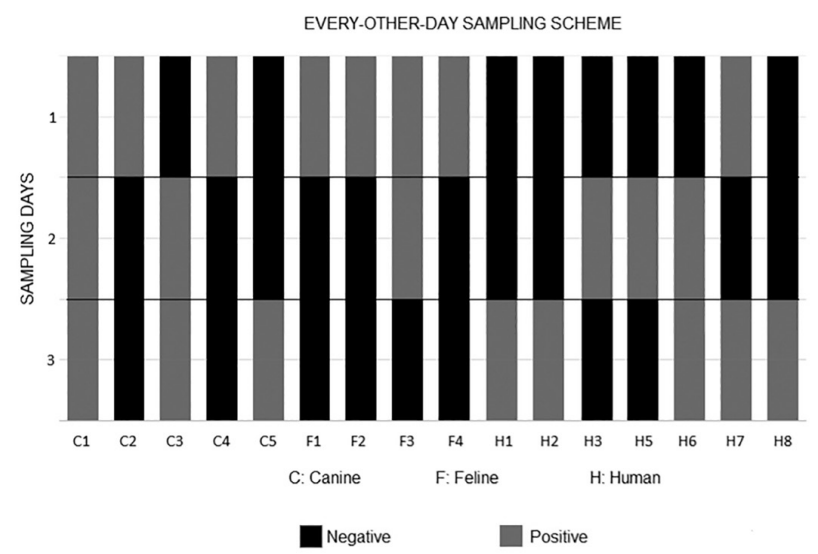

Figure 1 - Chart showing the detection of Giardia duodenalis cysts in stool samples from all the three animal species assessed in the present study (humans, dogs, and cats), and the number of fecal specimens that were collected during the daily sampling scheme and examined using the Faust technique ${ }^{1}$

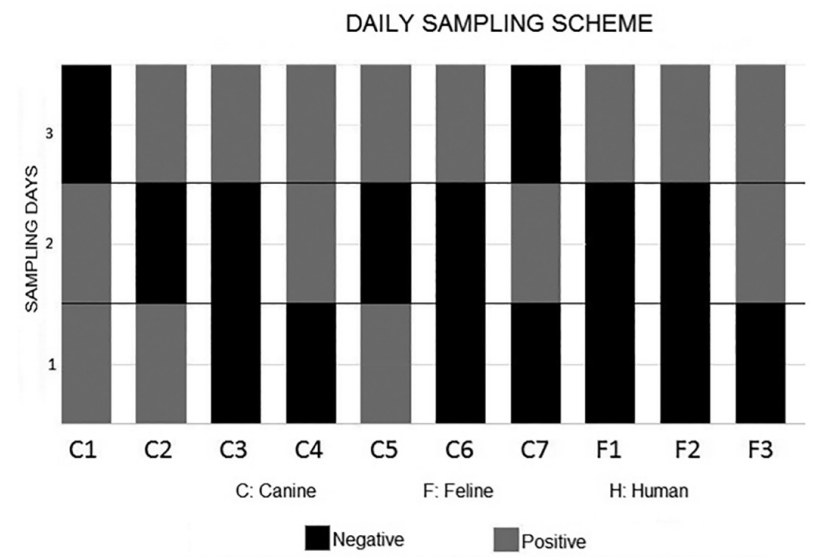

Figure 2 - Chart showing the detection of Giardia duodenalis cysts in stool samples from all the three animal species assessed in the present study (humans, dogs, and cats), and the number of fecal specimens that were collected during the every-other-day sampling scheme and examined using the Faust technique ${ }^{1}$

and, with the collection of three samples, 4 more Giardiapositive animals were diagnosed, with an increase of $16.7 \%(2 / 12)(\mathrm{p}=0.44)$ and $33.3 \%(4 / 12)(\mathrm{p}=0.09)$ in the detection of carriers, respectively. When comparing one-day

Table 2 - Number of Giardia duodenalis-positive patients considering the number of samples collected in each sampling scheme and in each animal species analyzed (humans, dogs, and cats). The Faust technique ${ }^{1}$ was used to screen fecal specimens

\begin{tabular}{lcccccc}
\hline & \multicolumn{5}{c}{ Giardia-positive samples } \\
\cline { 2 - 7 } & \multicolumn{5}{c}{ Daily sampling } & \multicolumn{3}{c}{ Every-other-day sampling } \\
\cline { 2 - 7 } & $\mathrm{S} 1$ & $\mathrm{~S} 1+2$ & $\mathrm{~S} 1+2+3$ & $\mathrm{~S} 1$ & $\mathrm{~S} 1+2$ & $\mathrm{~S} 1+2+3$ \\
\hline Humans & 0 & 0 & 0 & $5(71.4 \%)$ & $7(100 \%)$ & $7(100 \%)$ \\
Dogs & $3(42.9 \%)$ & $5(71.4 \%)$ & $7(100 \%)$ & $3(60 \%)$ & $3(60 \%)$ & $5(100 \%)$ \\
Cats & 0 & $1(33.3 \%)$ & $3(100 \%)$ & 0 & $1(25 \%)$ & $4(100 \%)$ \\
All three species & $3(30 \%)$ & $6(60 \%)$ & $10(100 \%)$ & $8(50 \%)$ & $11(68.7 \%)$ & $16(100 \%)$ \\
\hline
\end{tabular}


sampling with three-day sampling, there was an increase of 6 Giardia-positive animals $(50 \%)(\mathrm{p}=0.01)$. In dogs, no significant difference between the two sampling schemes was observed $(\mathrm{p}=0.99)$.

The collection of two and three samples in the daily collection scheme provided two more positive animals each, increasing the positivity by $28.6 \%$ in the each extra sampling day scheme ( $\mathrm{p}=0.59$ and $\mathrm{p}=0.46$, respectively). Regarding the every-other-day scheme, there was an increase of 2 positive animals only with the collection of three samples, rising the positivity by $40 \%(\mathrm{p}=0.44)$.

An increase in the diagnostic sensitivity when multiple stool samples were examined for the presence of Giardia cysts was particularly relevant in cats: $2(28.6 \%)$ of them were diagnosed with two collected samples $(\mathrm{p}=0.46)$ and the other $5(71.4 \%)$ with three collected samples $(\mathrm{p}=0.02)$. No cats were diagnosed with a single sample collection. When comparing the two sampling schemes in feline samples, there was a decrease of $35.0 \%$ in the number of positive fecal specimens when using the every-other-day scheme $(\mathrm{p}=0.28)$. Taking into account the feline samples, it was observed that positive cats were diagnosed only with two and three collected samples, in both schemes. In the daily scheme, a percentage increase of $33.3(\mathrm{p}=1.00)$ and $66.7(\mathrm{p}=0.40)$ in each extra day of collection was observed, respectively. When samples were collected alternately, increases of $25 \%$ on the second $(\mathrm{p}=1.00)$ and $75 \%$ on the third day $(\mathrm{p}=0.14)$ of sampling collection were observed. When comparing the single sample collection with the three-day sample collection, there was a significant difference favoring the every-other-day sampling scheme $(\mathrm{p}=0.02)$.

When considering fecal samples from all the three species studied and from both sampling groups, there was an increase of 6 Giardia-positive patients with the two-day sampling and 9 Giardia-positive patients with the threeday sampling with an increase of $23.1 \%(\mathrm{p}=0.10)$ and $34.6 \%(0.001)$ in the detection of carriers of this protozoan infection, respectively. When the two serial sampling schemes were compared in all animal species, there was an increase of $13.6 \%$ in the number of Giardia-positive fecal specimens if those were sampled by the every other day scheme $(\mathrm{p}=0.07)$.

Taking into account all three species, when stool samples were collected daily, the second and the third sample collection afforded the diagnosis of, respectively, more three and seven patients in comparison with the single collection scheme, raising the number of positive cases by $30 \%(\mathrm{p}=0.21)$ and $70 \%(\mathrm{p}=0.003)$. In the every-other-day scheme, it was possible to identify three more patients with two collected samples $(18.7 \% ; \mathrm{p}=0.47)$ and eight more patients with three collected samples $(50 \%$; $\mathrm{p}=0.002)$.
Stool samples that were Giardia-positive in one assessment were Giardia-negative in other assessment; this pattern was observed in $100 \%$ of humans (7/7), $91.7 \%$ of dogs (11/12) and $100 \%$ of cats (7/7) and was present in 25 (96.2\%) of 26 positive patients.

In the group in which fecal specimens were sampled on consecutive days (daily sampling), intermittent cyst shedding was observed in $100 \%$ of dogs and cats (Figure 1). In the group assigned to collect fecal samples every other day (every-other-day sampling), intermittent excretion of protozoan cysts in stool was observed in 100\% of humans (7/7), $80 \%$ of the dogs (4/5) and $100 \%$ of cats (4/4). In that group, $93.7 \%$ of patients $(15 / 16)$ that were positive for Giardia presented intermittent cyst shedding (Figure 2).

\section{DISCUSSION}

Since the same number of stool sample kits was distributed among individuals from each sampling group assessed, the difference in the number of fecal samples collected by each sampling group was due to the fact that some of the participants didn't collect samples every other day as requested. A major drawback in parasitology research involving fecal specimen collection by volunteers is that there are participants who fail to follow the provided instruction ${ }^{27}$. In the present study, serial sampling of multiple specimens by many individuals was an additional confounding factor.

In this study, multiple fecal sampling accounted for an increase in the number of Giardia-positive fecal samples in all three species examined and in both sampling schemes. Other authors that also assessed the influence of serial sampling on the diagnosis of giardiasis in humans ${ }^{13,16,21,28,29}$ and dogs ${ }^{18,19,30,31}$ reported similar findings. Since no studies were observed in a comprehensive literature search, to our knowledge, this is the first study assessing the influence of serial sampling on feline hosts.

Contrary to our findings, Györkös et al. ${ }^{32}$, Senay and MacPherson ${ }^{33}$, and Morris et al. ${ }^{34}$ reported no significant increase in the number of positive samples for intestinal parasites detection when the serial sampling scheme was used. It is important to highlight that not all of those authors studied the use of serial sample collection for diagnosing protozoa. Györkös et al.$^{32}$ performed serial fecal examination for intestinal parasites detection only in asymptomatic human carriers, and Senay and MacPherson ${ }^{33}$, and Morris et al.$^{34}$ tested stool samples from symptomatic human patients for enteric parasites detection. Moreover, the methods used for fecal examination and sample preservation were not standardized in these studies. 
Senay and MacPherson ${ }^{33}$ used the concentration formalinether technique on sodium acetate-formalin preserved samples; Morris et al. ${ }^{34}$ used the concentration by formalinethyl acetate method on 10\% formalin and PVA preserved samples; and Györkös et al. . $^{32}$ used the Faust and formalinether concentration techniques on sodium acetate-formalin preserved samples. It is worth noting that the authors were not expecting to specifically diagnose Giardia duodenalis infection so that a more sensitive technique for this parasite diagnosis was not used to analyze the samples. Many of intestinal parasites don't have an intermittently shedding of ova or cysts like Giardia sp. Nevertheless, it should be emphasized that those studies were conducted in developed countries, in areas where the prevalence of Giardia is low whereas our study was conducted in a developing country (Brazil). Since epidemiological and socioeconomic features in each of these geographical areas vary significantly, different results are expected in similar assessment studies performed in each of those regions.

Smith and Wolfe ${ }^{35}$ reported that the use of triplicate fecal samples for the diagnosis of giardiasis would decrease the chance of a false negative result that would occur due to intermittent cyst excretion in humans. After that, over the years, that feature was reaffirmed by other authors, who examined the presence of Giardia cysts in infected dogs by serial evaluation of fecal samples ${ }^{18,19,31}$. This finding is in agreement with the findings from our study, where the excretion of G. duodenalis cysts in the feces of $92.6 \%$ of positive volunteers was intermittent in both sampling groups and in patients from all species. The increase of Giardiapositive fecal specimens in the every-other-day sampling scheme can also be attributed to the aforementioned intermittent cyst shedding pattern of $G$. duodenalis.

Fecal sampling has proven to be an issue in research studies about intestinal parasites in all types of hosts ${ }^{36,37}$. With regard to pets (dogs and cats), owners may be unwilling to cooperate in research and would refuse to sample fecal specimens from their animals and send those samples for parasitological examination as the odor and appearance of those stools would repulse them. In addition, the patient identification and estimating defecation time and rate can also be difficult especially in environments inhabited by many animals from different species, in particular in households with large outdoor areas that are shared by dogs and cats. Thus, the correct instruction for volunteers about sampling methods and the awareness of the pet owners about the importance of multiple fecal exams are important to ensure reliable results. Therefore, albeit sampling problems are possible in a methodology based on volunteers' collection of samples, actions involving volunteer instructions were taken to minimize the influence of the aforementioned problems on the results.

Despite the limited number of fecal samples from each species that was analyzed in the present study, to the best of the authors' knowledge, there are no previous studies published to date with similar methodology for sample collection (consecutive versus every-other-day) involving all three species studied.

\section{CONCLUSIONS}

Based on the results of this study, we conclude that multiple fecal sampling increases the sensitivity and accuracy of the Faust et al..$^{25}$ technique to diagnose G. duodenalis infection in the three animal species examined, regardless of the sampling scheme. This procedure increases the number of tested samples but overcomes the interference of the intermittent excretion of Giardia cysts.

\section{REFERENCES}

1. Flanagan PA. Giardia-diagnosis, clinical course and epidemiology. A review. Epidemiol Infect. 1992;109:1-22.

2. Thompson RC. The zoonotic significance and molecular epidemiology of Giardia and Giardiasis. Vet Parasitol. 2004;126:15-35.

3. Esch KJ, Petersen CA. Transmission and epidemiology of zoonotic protozoal diseases of companion animals. Clin Microbiol Rev. 2013;26:58-85

4. Thompson RC, Palmer CS, O'Handley R. The public health and clinical significance of Giardia and Cryptosporidium in domestic animals. Vet J. 2008;177:18-25.

5. Danciger M, Lopez M. Numbers of Giardia in the feces of infected children. Am J Trop Med Hyg. 1975;24:237-42.

6. Zimmer JF, Burrington DB. Comparison of four techniques of fecal examination for detecting canine giardiasis. J Am Anim Hosp Assoc. 1986;22:161-7.

7. Ortega YR, Adam RD. Giardia: overview and update. Clin Infect Dis. 1997;25:545-9.

8. Cama VA, Mathison BA. Infections by intestinal coccidia and Giardia duodenalis. Clin Lab Med. 2015;35:423-44.

9. Conboy G. Giardia. Can Vet J. 1997;38:245-7.

10. Koehler AV, Jex AR, Haydon SR, Stevens MA, Gasser RB. Giardia/giardiasis - a perspective on diagnostic and analytical tools. Biotechnol Adv. 2014;32:280-9.

11. Zimmerman SK, Needham CA. Comparison of conventional stool concentration and preserved-smear methods with Merifluor Cryptosporidium/Giardia Direct Immunofluorescence Assay and ProSpecT giardia EZ Microplate Assay for detection of Giardia lamblia. J Clin Microbiol. 1995;33:1942-3. 
12. Stojecki K, Sroka J, Karamon P, Kusyk T, Cencek T. Influence of selected stool concentration techniques on the effectiveness of PCR examination in Giardia intestinalis diagnostics. Pol J Vet Sci. 2014;17:19-25.

13. Cartwright CP. Utility of multiple-stool-specimen ova and parasite examinations in a high-prevalence setting. J Clin Microbiol. 1999;37:2408-11.

14. Schuurman T, Lankamp P, van Belkum A, Kooistra-Smid M, van Zwet A. Comparison of microscopy, real-time PCR and a rapid immunoassay for the detection of Giardia lamblia in human stool specimens. Clin Microbiol Infect. 2007;13:1186-91.

15. Kirkpatrick CE, Farrell JP. Feline giardiasis: observations on natural and induced infections. Am J Vet Res. 1984;45:2182-8.

16. Heymans HS, Aronson DC, van Hooft MA. Giardiasis in childhood: an unnecessarily expensive diagnosis. Eur J Pediatr. 1987;146:401-3.

17. Garcia LS, Shimizu RY. Evaluation of nine immunoassay kits (enzyme immunoassay and direct fluorescence) for detection of Giardia lamblia and Cryptosporidium parvum in human fecal specimens. J Clin Microbiol. 1997;35:1526-9.

18. Decock C, Cadiergues MC, Larcher M, Vermot S, Franc M. Comparison of two techniques for diagnosis of giardiasis in dogs. Parasite. 2003;10:69-72.

19. Dryden MW, Payne PA, Smith V. Accurate diagnosis of Giardia spp and proper fecal examination procedures. Vet Ther. 2006;7:4-14.

20. Staat MA, Rice M, Donauer S, Mukkada S, Holloway M, Cassedy A, et al. Intestinal parasite screening in internationally adopted children: importance of multiple stool specimens. Pediatrics. 2011;128:e613-22.

21. Duffy TL, Montenegro-Bethancourt G, Solomons NW, Belosevic M, Clandinin MT. Prevalence of giardiasis in children attending semi-urban daycare centres in Guatemala and comparison of 3 Giardia detection tests. J Health Popul Nutr. 2013;31:290-3.

22. Sadaka HA, Gaafar MR, Mady RF, Hezema NN. Evaluation of ImmunoCardSTAT test and ELISA versus light microscopy in diagnosis of giardiasis and cryptosporidiosis. Parasitol Res. 2015;114:2853-63.

23. Gates MC, Nolan TJ. Comparison of passive fecal flotation run by veterinary students to zinc-sulfate centrifugation flotation run in a diagnostic parasitology laboratory. J Parasitol. 2009;95:1213-4.
24. Hanson KL, Cartwright CP. Use of an enzyme immunoassay does not eliminate the need to analyze multiple stool specimens for sensitive detection of Giardia lamblia. J Clin Microbiol. 2001;39:474-7.

25. Faust EC, Sawitz W, Tobie J, Odom V, Peres C, Lincicome DR. Comparative efficiency of various technics for the diagnosis of protozoa and helminths in feces. J Parasitol. 1939;25:241-62.

26. Pereira DS, Ferreira CS. Método de Faust et al.: rendimento de colheita por alça metálica. Rev Inst Med Trop São Paulo. 1991;33:153-8.

27. Mosli M, Gregor J, Chande N, Lannigan R. Nonutility of routine testing of stool for ova and parasites in a tertiary care Canadian centre. Can J Microbiol. 2012;58:653-9.

28. Adam RD. The biology of Giardia sp. Microbiol Rev. 1991;55:706-32.

29. Hiatt RA, Markell, EK, Ng E. How many stool examinations are necessary to detect pathogenic intestinal protozoa? Am J Trop Med Hyg. 1995;53:36-9.

30. Jacobs SR, Forrester CP, Yang J. A survey of the prevalence of Giardia in dogs presented to Canadian veterinary practices. Can Vet J. 2001;42:45-6.

31. Rishniw M, Liotta J, Bellosa M, Bowman D, Simpson KW. Comparison of 4 Giardia diagnostic tests in diagnosis of naturally acquired canine chronic subclinical giardiasis. J Vet Intern Med. 2010;24:293-7.

32. Györkös TW, MacLean JD, Law CG. Absence of significant differences in intestinal parasite prevalence estimates after examination of either one or two stools specimens. Am J Epidemiol. 1989;130:976-80.

33. Senay H, MacPherson D. Parasitology: diagnostic yield of stool examination. CMAJ. 1989;140:1329-31.

34. Morris AJ, Wilson ML, Reller LB. Application of rejection criteria for stool ovum and parasite examinations. J Clin Microbiol. 1992;30:3213-6.

35. Smith JW, Wolfe MS. Giardiasis. Annu Rev Med. 1980;31:373-83.

36. Mohammed Mahdy AK, Lim YA, Surin J, Wan KL, Al-Mekhlafi MS. Risk factors for endemic giardiasis: highlighting the possible association of contaminated water and food. Trans $\mathrm{R}$ Soc Trop Med Hyg. 2008;102:465-70.

37. Katagiri S, Oliveira-Sequeira TC. Comparison of three concentration methods for the recovery of canine intestinal parasites from stool samples. Exp Parasitol. 2010;126:214-6. 